\title{
On symmetric logarithm and some old examples in smooth ergodic theory
}

\author{
by
}

\author{
K. Frączek and M. Lemańczyk (Toruń)
}

\begin{abstract}
We give a positive answer to the problem of existence of smooth weakly mixing but not mixing flows on some surfaces. More precisely, on each compact connected surface whose Euler characteristic is even and negative we construct smooth weakly mixing flows which are disjoint in the sense of Furstenberg from all mixing flows and from all Gaussian flows.
\end{abstract}

1. Introduction. Smooth flows preserving a smooth positive measure on a compact connected surface are object of interest in this paper. More precisely, we will deal with the realization problem, i.e. with the problem of existence of smooth, flows on surfaces satisfying some prescribed ergodic properties. For the background on the realization problem we refer to $[10$, Part III]. The problem of existence of ergodic flows on surfaces has been solved by Blokhin in [4]. He constructed examples of ergodic $C^{\infty}$-flows on all surfaces, except the sphere, the projective plane, and the Klein bottle on which there are no such flows. For the first two surfaces the absence of ergodic smooth flows follows from the Poincaré-Bendixson theorem. The absence of ergodic smooth flows on the Klein bottle was proved by Aranson [3] and Markley [19]. Moreover, as shown by Kochergin in [14], on any surface different from the sphere, the projective plane, and the Klein bottle, there is a mixing $C^{\infty}$-flow preserving a positive $C^{\infty}$-measure.

The problem of whether there exist smooth weakly mixing but not mixing flows on any surface is of certain interest (see [10, Part III]). In the case where the dimension of a manifold is at least 3, the positive solution of this problem has been given by Anosov and Katok in [2]. Our purpose is to give a positive partial answer in the two-dimensional case. The existence of smooth weakly mixing but not mixing flows on the torus is well known. In

2000 Mathematics Subject Classification: 37A10, 37E35, 37C40.

Research partly supported by KBN grant 1 P03A 03826 . 
this paper we will construct weakly mixing but not mixing smooth flows on any compact connected smooth surface whose Euler characteristic is even and negative. In fact, we will show that such flows can be found in the class of flows constructed by Blokhin in [4]. More precisely, we will deal with flows which can be represented as special flows over irrational rotations with a "symmetric logarithm form" (up to an absolutely continuous function) of the ceiling function. In Section 4, we prove that such flows are weakly mixing (the absence of mixing has been proved in [17]). Moreover, we will compute some limit distributions of a cocycle given by the ceiling function. These limit distributions, by [6], give rise to some strong joining properties of such flows. We will conclude that our examples are disjoint from all mixing and all Gaussian flows (in fact, from all ELF flows, see [6]) and are spectrally disjoint from all mixing flows, whenever the rotation in the base admits a sufficiently fast approximation by rational numbers. This will allow us to prove the following.

TheOREm 1. For any compact connected $C^{\infty}$-surface $M$ with Euler characteristic $\chi=-2 k, k \geq 1$, there exists a positive $C^{\infty}$-measure-preserving $C^{\infty}$-flow on $M$ which is

- weakly mixing,

- disjoint in the sense of Furstenberg from all Gaussian flows,

- spectrally disjoint from all mixing flows.

2. Basic definitions and notation. Assume that $T:(X, \mathcal{B}, \mu) \rightarrow$ $(X, \mathcal{B}, \mu)$ is an ergodic automorphism of a standard probability space. We will also denote by $T$ the associated unitary action

$$
f \mapsto f \circ T
$$

on the subspace $L_{0}^{2}(X, \mathcal{B}, \mu)$ of all zero-mean functions of $L^{2}(X, \mathcal{B}, \mu)$. A measurable function $f: X \rightarrow \mathbb{R}$ determines a cocycle $f^{(\cdot)}(\cdot): \mathbb{Z} \times X \rightarrow \mathbb{R}$ given by

$$
f^{(m)}(x)= \begin{cases}f(x)+f(T x)+\ldots+f\left(T^{m-1} x\right) & \text { if } m>0, \\ 0 & \text { if } m=0, \\ -\left(f\left(T^{m} x\right)+\ldots+f\left(T^{-1} x\right)\right) & \text { if } m<0 .\end{cases}
$$

We denote by $T_{f}$ the corresponding cylinder flow acting on $(X \times \mathbb{R}, \mathcal{B} \otimes$ $\mathcal{B}(\mathbb{R}), \mu \otimes \lambda)(\lambda$ stands for Lebesgue measure on $\mathbb{R})$ by the formula

$$
T_{f}(x, r)=(T x, f(x)+r) .
$$

By $\left(f^{(n)}\right)_{*} \mu$ we will mean the measure on $\mathbb{R}$ which is the image of $\mu$ via $f^{(n)}$. Therefore $\left(f^{(n)}\right)_{*} \mu \in \mathcal{P}(\mathbb{R})$, where $\mathcal{P}(\mathbb{R})$ denotes the space of all probability 
Borel measures on $\mathbb{R}$. By $\lambda_{\mathbb{T}}$ we will denote Lebesgue measure on the circle $\mathbb{T}=\mathbb{R} / \mathbb{Z}$, which we will constantly identify with $[0,1)$.

If $g: X \rightarrow \mathbb{R}$ is a strictly positive $L^{1}$-function, then we denote by $T^{g}=$ $\left(T_{t}^{g}\right)_{t \in \mathbb{R}}$ the corresponding special flow under $g$ (see e.g. [5, Chapter 11]) acting on $\left(X^{g}, \mathcal{B}^{g}, \mu^{g}\right)$, where $X^{g}=\{(x, s) \in X \times \mathbb{R}: 0 \leq s<g(x)\}$ and $\mathcal{B}^{g}$ (resp. $\mu^{g}$ ) is the restriction of $\mathcal{B} \otimes \mathcal{B}(\mathbb{R})$ (resp. $\mu \otimes \lambda$ ) to $X^{g}$. Under the action of the flow $T^{g}$ each point in $X^{g}$ moves vertically at unit speed, and we identify the point $(x, g(x))$ with $(T x, 0)$.

Two flows $T^{(i)}=\left(\left(T^{(i)}\right)_{t}\right)_{t \in \mathbb{R}}$ that act on $\left(X^{(i)}, \mathcal{B}^{(i)}, \mu^{(i)}\right), i=1,2$, are said to be disjoint in the sense of Furstenberg (see [7]) if the only $\left(\left(T^{(1)}\right)_{t} \times\right.$

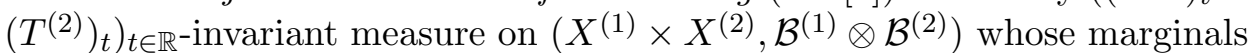
are $\mu^{(1)}$ and $\mu^{(2)}$ resp. is the product measure. Recall that $T^{(1)}$ and $T^{(2)}$ are spectrally disjoint if the maximal spectral types of $T^{(1)}$ and $T^{(2)}$ are mutually singular.

If $\alpha \in \mathbb{T}$ is an irrational number, then we denote by $T x=x+\alpha$ the corresponding irrational rotation on $\left(\mathbb{T}, \mathcal{B}(\mathbb{T}), \lambda_{\mathbb{T}}\right)$. For a real number $t$ denote by $\{t\}$ its fractional part and by $\|t\|$ its distance to the nearest integer. We have $\|-t\|=\|t\|$ and $\left\|t_{1}+t_{2}\right\| \leq\left\|t_{1}\right\|+\left\|t_{2}\right\|$. For an irrational $\alpha \in \mathbb{T}$ denote by $\left(q_{n}\right)$ its sequence of denominators (see e.g. [11]), that is, we have

where

$$
\left|\alpha-\frac{p_{n}}{q_{n}}\right|<\frac{1}{q_{n}^{2}}
$$

$$
\begin{array}{ll}
q_{0}=1, & q_{1}=a_{1}, \quad q_{n+1}=a_{n+1} q_{n}+q_{n-1}, \\
p_{0}=0, & p_{1}=1, \quad p_{n+1}=a_{n+1} p_{n}+p_{n-1},
\end{array}
$$

and $\left[0 ; a_{1}, a_{2}, \ldots\right]$ stands for the continued fraction expansion of $\alpha$.

3. Blokhin's examples. In this section we briefly recall some examples of ergodic flows which preserve positive smooth measures on surfaces constructed by Blokhin in [4]. More precisely, we will deal with compact connected smooth surfaces whose Euler characteristic is even and negative. Applying Kochergin's arguments (see [15]), we will deduce that some of the flows can be represented as special flows built over irrational rotations on the circle and under functions which are sums of the symmetric logarithm (i.e. $-(\log \{x\}+\log \{-x\}))$ and absolutely continuous functions.

Let us consider the flow $\left(\varphi_{t}\right)_{t \in \mathbb{R}}$ on the plane given by the Hamiltonian $H(x, y)=y\left(x^{2}+y^{2}-1\right)$. Let $R$ be the ring on the plane bounded by the circles $S_{i}=\left\{(x, y): x^{2}+y^{2}=r_{i}^{2}\right\}, i=1,2$, where $1 / \sqrt{3}<r_{1}<1<r_{2}$. Then the unit circle consists of two saddle points $A=(-1,0), B=(1,0)$ and two separatrices (see Fig. 1).

We first present examples of required flows on the smooth surfaces with $\chi=-2$. Let $\alpha$ be an irrational number. 


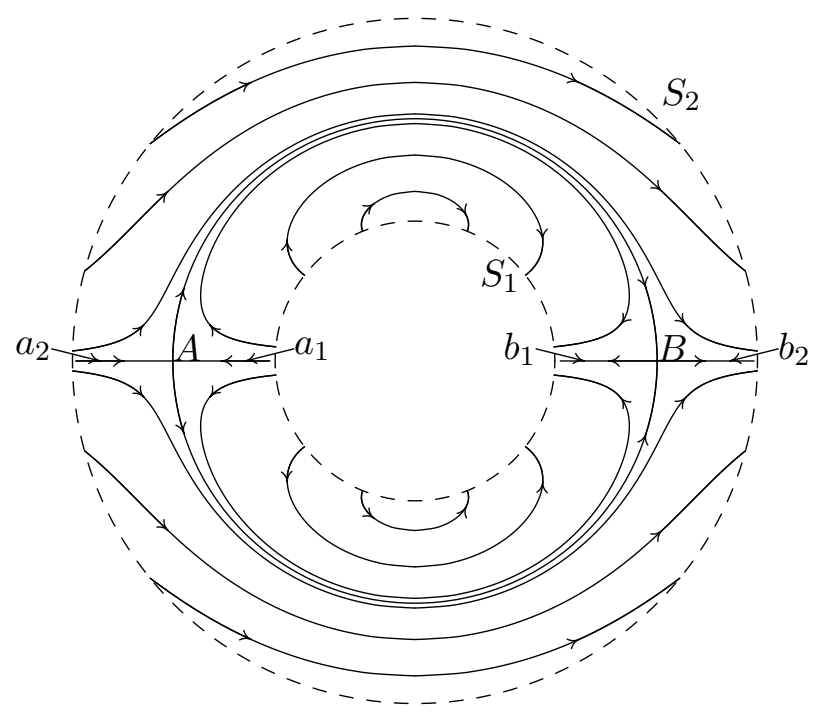

Fig. 1

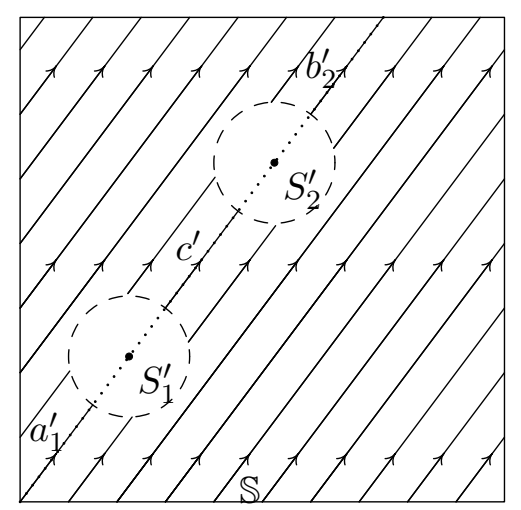

Fig. 2

We denote by $\left(\psi_{t}\right)_{t \in \mathbb{R}}$ the irrational rotation $\psi_{t}\left(x_{1}, x_{2}\right)=\left(x_{1}+t \alpha, x_{2}+t\right)$ of the torus $\mathbb{T}^{2}$. Let us cut out two disjoint disks $D_{1}, D_{2}$ from the torus which are disjoint from the circle $\mathbb{S}=\left\{(x, 0) \in \mathbb{T}^{2}: x \in \mathbb{R}\right\}$ and whose centers lie on the segment $O_{[0,1]}=\left\{\psi_{t}(0,0): t \in[0,1]\right\}$ (see Fig. 2). We will denote by $S_{1}^{\prime}$ and $S_{2}^{\prime}$ the circles which bound $D_{1}$ and $D_{2}$. Then the set $O_{[0,1]} \backslash\left(D_{1} \cup D_{2}\right)$ consists of three segments $a_{1}^{\prime}, c^{\prime}, b_{2}^{\prime}$ of the positive orbit of $(0,0)$. Now one can construct a $C^{\infty}$-diffeomorphism $f: S_{1} \cup S_{2} \rightarrow S_{1}^{\prime} \cup S_{2}^{\prime}$ such that

- the flows $\left(\psi_{t}\right)_{t \in \mathbb{R}}$ on $\mathbb{T}^{2} \backslash\left(D_{1} \cup D_{2}\right)$ and $\left(\varphi_{t}\right)_{t \in \mathbb{R}}$ on $R$ are glued through $f$ into a $C^{\infty}$-flow $\left(\psi_{t}^{\prime}\right)_{t \in \mathbb{R}}$ on the $C^{\infty}$-surface $\left(\mathbb{T}^{2} \backslash\left(D_{1} \cup D_{2}\right)\right) \cup_{f} R$ which preserves a positive $C^{\infty}$-measure, 
- the segment $O_{[0,1]}$ is glued with the separatrices of the saddle points on $R$; more precisely the end of $a_{1}^{\prime}$ is glued with the beginning of $a_{1}$, the end of $b_{1}$ with the beginning $c^{\prime}$, the end of $c^{\prime}$ with the beginning of $a_{2}$, and the end of $b_{2}$ with the beginning of $b_{2}^{\prime}$,

- for every $x \in \mathbb{S} \backslash(0,0)$ the positive orbits of $x$ for the flows $\left(\psi_{t}\right)_{t \in \mathbb{R}}$ and $\left(\psi_{t}^{\prime}\right)_{t \in \mathbb{R}}$ agree on the set $\mathbb{T}^{2} \backslash\left(D_{1} \cup D_{2}\right)$.

For more details of the construction we refer the reader to [4]. It is clear that Blokhin's construction leads to the flow on the pretzel or on the nonorientable surface with $\chi=-2$. Moreover, the circle $\mathbb{S}$ is a transversal curve for $\left(\psi_{t}^{\prime}\right)_{t \in \mathbb{R}}$ such that with the exception of the saddle separatrices (which form a set of zero measure) all points from the surface lie on positive orbits which start from $\mathbb{S}$. Of course, the return map on $\mathbb{S}$ is rotation by $\alpha$ and moreover the return time map is of class $C^{\infty}$ on $\mathbb{S} \backslash(0,0)$. Consequently, $\left(\psi_{t}^{\prime}\right)_{t \in \mathbb{R}}$ is measure-theoretically isomorphic to a special flow $T^{f}$, where $T$ : $\mathbb{T} \rightarrow \mathbb{T}, T x=x+\alpha$ and $f: \mathbb{T} \rightarrow \mathbb{R}$ is a positive function with finite integral; moreover, $f$ is of class $C^{\infty}$ on $\mathbb{T} \backslash\{0\}$. On the other hand, by the proof of Lemma 3.3 in [15] and arguments presented before this lemma, we can conclude that $f(x)=-a \log |x|+g(x)$ on an interval $(-\varepsilon, \varepsilon)$, where $a>0$ and $g:(-\varepsilon, \varepsilon) \rightarrow \mathbb{R}$ is a continuous function of class $C^{\infty}$ on $(-\varepsilon, \varepsilon) \backslash\{0\}$ and such that $g^{\prime} \in L^{1}([-\varepsilon, \varepsilon])$. Consequently, there exists a continuous function $h: \mathbb{T} \rightarrow \mathbb{R}$ which is of class $C^{\infty}$ on $\mathbb{T} \backslash\{0\}$, such that $h^{\prime} \in L^{1}(\mathbb{T})$ and $f(x)=-a(\log \{x\}+\log \{-x\})+h(x)$. Then the function $\bar{h}:[0,1] \rightarrow \mathbb{R}$ given by $\bar{h}(x)=\int_{0}^{x} h^{\prime}(t) d t+h(0)$ is absolutely continuous and

$$
\bar{h}(x)=\lim _{y \rightarrow 0+} \int_{y}^{x} h^{\prime}(t) d t+h(0)=\lim _{y \rightarrow 0+}(h(x)-h(y))+h(0)=h(x),
$$

whenever $x \in(0,1)$. By the continuity of $h$, we conclude that $h=\bar{h}$, and finally that $h: \mathbb{T} \rightarrow \mathbb{R}$ is absolutely continuous. Now $k$-fold repetition of the above procedure of gluing of the flow $\left(\varphi_{t}\right)_{t \in \mathbb{R}}$ to the flow $\left(\psi_{t}\right)_{t \in \mathbb{R}}$ (in the orientable or nonorientable way) allows us to formulate the following.

Proposition 2. For any irrational $\alpha$ and any compact connected $C^{\infty}$ surface $M$ with $\chi=-2 k, k \geq 1$, one can find a $C^{\infty}$-flow on $M$ which:

- has an invariant positive $C^{\infty}$-measure,

- is measure-theoretically isomorphic to a special flow $T^{f}$ built over the rotation $T x=x+\alpha$ and under a function $f(x)=-a(\log \{x\}+\log \{-x\})+$ $h(x)$, where $a>0$ and $h: \mathbb{T} \rightarrow \mathbb{R}$ is an absolutely continuous function.

4. Some properties of cocycles with a single logarithmic singularity. Let us consider $f \in L^{1}(\mathbb{T})$ with $\int f(x) d x=0$ satisfying the following conditions: 


$$
\begin{gathered}
f \in C^{1}(0,1), \\
f^{\prime} \text { is monotonic, } \\
\lim _{x \rightarrow 0^{+}} x f^{\prime}(x)=c, \quad \text { where } c \neq 0, \\
\operatorname{Re} \widehat{f}[n]=O(1 / n) .
\end{gathered}
$$

Let $T x=x+\alpha$ be an irrational rotation on the circle. Denote by $\left(q_{n}\right)$ the sequence of denominators of $\alpha$. By passing to a further subsequence if necessary we can assume that

$$
\left|\alpha-\frac{p_{n}}{q_{n}}\right| \leq \frac{1}{\sqrt{5} q_{n}^{2}}
$$

(the existence of such a subsequence is shown e.g. in [11]). Set $\widetilde{f}(x)=$ $f(x)+f(-x), x \in \mathbb{T}$. Then $\widehat{\widetilde{f}}[n]=\widehat{f}[n]+\widehat{f}[-n]=2 \operatorname{Re} \widehat{f}[n]$ and consequently $($ see $(4))$

$$
\widehat{\widetilde{f}}[n]=O(1 / n) .
$$

Proposition 3. There exist $s_{0}>0$ and $0<\eta<1$ such that

$$
\limsup _{n}\left|\int_{\mathbb{T}} e^{2 \pi i s \tilde{f}^{\left(q_{n}\right)}(x)} d x\right| \leq \eta
$$

whenever $|s| \geq s_{0}$.

Proof. By passing to a further subsequence we can assume that all $q_{n}$ are even denominators of $\alpha$, i.e. $\alpha>p_{n} / q_{n}$ (the reasoning in case $\alpha<p_{n} / q_{n}$ is similar). Fix $n \in \mathbb{N}$ and put $q:=q_{n}$. For $j=0, \ldots, q-1$ set

$$
a_{j}=\frac{j}{q}+\frac{1}{100 q}, \quad b_{j}=a_{j}+\frac{1}{100 q}, \quad c_{j}=\frac{j+1}{q}-\frac{1}{50 q}, \quad d_{j}=c_{j}+\frac{1}{100 q}
$$

and let $A_{q}=\bigcup_{j=0}^{q-1}\left[a_{j}, b_{j}\right]$. Then

$$
\left|A_{q}\right|=\frac{1}{100} .
$$

Take $\Delta=1 / 2-(1 / 50+1 / \sqrt{5})>0$. Now observe that if $x \in A_{q}$, then $x, x+p / q, \ldots, x+(q-1) p / q(\bmod 1)$ do not leave $A_{q}$. Moreover, from (5), for each $j=0, \ldots, q-1$ we have

$$
\left|x+j \frac{p}{q}-(x+j \alpha)\right| \leq j\left|\alpha-\frac{p}{q}\right| \leq \frac{1}{\sqrt{5} q} .
$$

It follows that the interval $\left[a_{k}, b_{k}\right]+j \alpha$ (which is contained in $\left[k_{j} / q,\left(k_{j}+1\right) / q\right]$ ) is to the right of $\left[a_{k_{j}}, b_{k_{j}}\right]$ and the distance between them is majorized by 
$1 /(\sqrt{5} q)$. Now consider the set $-A_{q}$. Then

$$
-A_{q}=\bigcup_{j=0}^{q-1}\left[c_{j}, d_{j}\right]
$$

and since $A_{q}$ is invariant under rotation by $p / q$, so is $-A_{q}$. By symmetry, the interval $\left[c_{k}, d_{k}\right]-j \alpha$ (which is contained in $\left[l_{j} / q,\left(l_{j}+1\right) / q\right]$ ) is to the left of $\left[c_{l_{j}}, d_{l_{j}}\right]$ and the distance between them is estimated by $1 /(\sqrt{5} q)$.

We now show that

$$
\left|\tilde{f}^{\prime}(q)(x)\right| \geq \frac{|c| \Delta}{1 / 4-\Delta^{2}} q \quad \text { for all } x \in A_{q} .
$$

In fact, for each $x \in A_{q}$ and each $j=0, \ldots, q-1$, in each interval $[j / q$, $(j+1) / q$ ] there exists a unique point of the form $x+k_{j} \alpha$ and a unique point of the form $-x-l_{j} \alpha$. Moreover,

$$
\left(-x-l_{j} \alpha\right)-\left(x+k_{j} \alpha\right) \geq 2 \Delta \frac{1}{q} .
$$

Now applying (2) we obtain

$$
\begin{aligned}
\left|\widetilde{f}^{\prime(q)}(x)\right| & =\left|f^{\prime(q)}(x)-f^{\prime(q)}(-x)\right|=\left|\sum_{j=0}^{q-1} f^{\prime}\left(x+k_{j} \alpha\right)-f^{\prime}\left(-x-l_{j} \alpha\right)\right| \\
& \geq\left|f^{\prime}\left(x+k_{0} \alpha\right)-f^{\prime}\left(-x-l_{0} \alpha\right)\right| \\
& \geq\left|f^{\prime}\left(\left(\frac{1}{2}-\Delta\right) \frac{1}{q}\right)-f^{\prime}\left(\left(\frac{1}{2}+\Delta\right) \frac{1}{q}\right)\right| .
\end{aligned}
$$

From (3) it follows that if $\varepsilon>0$ is small enough, then

$$
\left|f^{\prime}\left(\left(\frac{1}{2}-\Delta\right) \frac{1}{q}\right)-f^{\prime}\left(\left(\frac{1}{2}+\Delta\right) \frac{1}{q}\right)\right| \geq \frac{(|c|-\varepsilon) q}{1 / 2-\Delta}-\frac{(|c|+\varepsilon) q}{1 / 2+\Delta}
$$

for all $q$ large enough, which gives (8).

Applying integration by parts and (8) we get

$$
\begin{aligned}
\left|\int_{a_{j}}^{b_{j}} e^{2 \pi i s \widetilde{f}^{(q)}(x)} d x\right| & =\frac{1}{2 \pi|s|}\left|\int_{a_{j}}^{b_{j}} \frac{1}{\widetilde{f}^{\prime(q)}(x)} d e^{2 \pi i s \widetilde{f}^{(q)}(x)}\right| \\
& =\frac{1}{2 \pi|s|}\left|\left[\frac{e^{2 \pi i s \widetilde{f}^{(q)}}}{\widetilde{f^{\prime}(q)}}\right]_{a_{j}}^{b_{j}}-\int_{a_{j}}^{b_{j}} e^{2 \pi i s \widetilde{f}^{(q)}(x)} d\left(\frac{1}{\widetilde{f^{\prime}(q)}(x)}\right)\right| \\
& \leq \frac{1}{2 \pi|s|}\left(\frac{2}{\min _{x \in\left[a_{j}, b_{j}\right]}\left|\widetilde{f}^{\prime(q)}(x)\right|}+\operatorname{Var}_{a_{j}}^{b_{j}}\left(\frac{1}{\widetilde{f}^{\prime(q)}}\right)\right) \\
& \leq \frac{1}{2 \pi|s|}\left(2 \delta \frac{1}{q}+\frac{\delta^{2}}{q^{2}} \operatorname{Var}_{a_{j}}^{b_{j}} \widetilde{f}^{\prime(q)}\right),
\end{aligned}
$$


where $\delta=\left(1 / 4-\Delta^{2}\right) /(|c| \Delta)$. Moreover,

$$
\begin{aligned}
\operatorname{Var}_{a_{j}}^{b_{j}} \tilde{f}^{\prime}(q) & =\operatorname{Var}_{a_{j}}^{b_{j}}\left(\widetilde{f}^{\prime}+\widetilde{f}^{\prime} \circ T+\ldots+\widetilde{f}^{\prime} \circ T^{q-1}\right) \\
& \leq \operatorname{Var}_{a_{j}}^{b_{j}} \widetilde{f}^{\prime}+\operatorname{Var}_{T a_{j}}^{T b_{j}}{\widetilde{f^{\prime}}}^{\prime}+\ldots+\operatorname{Var}_{T^{q-1} a_{j}}^{T^{q-1} b_{j}} \widetilde{f}^{\prime} .
\end{aligned}
$$

The intervals

$$
\begin{gathered}
{\left[a_{j}, b_{j}\right],\left[T a_{j}, T b_{j}\right], \ldots,\left[T^{q-1} a_{j}, T^{q-1} b_{j}\right]} \\
{\left[-a_{j},-b_{j}\right],\left[T^{-1}\left(-a_{j}\right), T^{-1}\left(-b_{j}\right)\right], \ldots,\left[T^{-(q-1)}\left(-a_{j}\right), T^{-(q-1)}\left(-b_{j}\right)\right]}
\end{gathered}
$$

are pairwise disjoint and the distance of their union from 0 (on the circle) is greater than $1 /(100 q)$. Consequently,

$$
\operatorname{Var}_{a_{j}}^{b_{j}} \widetilde{f}^{\prime(q)} \leq \operatorname{Var}_{\frac{1}{100 q}}^{\frac{99}{100}} \widetilde{f}^{\prime} \leq 2 \operatorname{Var}_{\frac{1}{100 q}}^{\frac{99}{100 q}} f^{\prime} .
$$

Now applying (2) and (3) gives

$$
\operatorname{Var}_{a_{j}}^{b_{j}} \tilde{f}^{\prime(q)} \leq 2\left|f^{\prime}\left(\frac{1}{100 q}\right)-f^{\prime}(1)\right| \leq 400|c| q
$$

for all $q$ large enough. It follows that

$$
\left|\int_{a_{j}}^{b_{j}} e^{2 \pi i s \widetilde{f}^{(q)}(x)} d x\right| \leq \frac{1}{2 \pi|s|}\left(2 \delta+400 \delta^{2}|c|\right) \frac{1}{q},
$$

and consequently that

$$
\left|\int_{A_{q}} e^{2 \pi i s \widetilde{f}^{(q)}(x)} d x\right| \leq \frac{1}{2 \pi|s|}\left(2 \delta+400 \delta^{2}|c|\right)=O(1 / s)
$$

for all $q$ large enough. Therefore by (7) we conclude that there exist $s_{0}>0$ and $0<\eta<1$ such that

$$
\left|\int_{\mathbb{T}} e^{2 \pi i s \widetilde{f}^{(q)}(x)} d x\right| \leq \eta
$$

whenever $|s| \geq s_{0}$ and $q$ is large enough.

Since the sequence $\left(\widetilde{f}^{\left(q_{n}\right)}\right)$ is bounded in $L^{2}(\mathbb{T})$ (see [1]), by passing to a further subsequence if necessary we can assume that the sequence of distributions $\left(\widetilde{f}^{\left(q_{n}\right)}\right)_{*} \lambda_{\mathbb{T}}$ satisfies

$$
\left(\widetilde{f}^{\left(q_{n}\right)}\right)_{*} \lambda_{\mathbb{T}} \rightarrow \varrho
$$

in the space $\mathcal{P}(\mathbb{R})$ of Borel probability measures on $\mathbb{R}$. By the proof of Proposition 12 in [18], we obtain the following conclusion.

COROLlary 4. The measure $\varrho$ is not a pure discrete measure and the cylinder flow

$$
T_{\widetilde{f}}:\left(\mathbb{T} \times \mathbb{R}, \lambda_{\mathbb{T}} \otimes \lambda\right) \rightarrow\left(\mathbb{T} \times \mathbb{R}, \lambda_{\mathbb{T}} \otimes \lambda\right), \quad T_{\widetilde{f}}(x, r)=(T x, \tilde{f}(x)+r),
$$

is ergodic. 
In addition to conditions (1)-(4) assume that $f$ is bounded from below. Let $C>0$ be a constant such that $\widetilde{f}+C>0$.

COROLlary 5. The special flow $T^{\tilde{f}+C}$ is weakly mixing and is disjoint from all mixing and all Gaussian flows.

Proof. The disjointness from all mixing and all Gaussian flows follows immediately from Corollaries 1 and 3 in [6]. Now suppose, contrary to our claim, that $T^{\tilde{f}+C}$ is not weakly mixing. Then there exist $s \geq s_{0}$ and a measurable function $g: \mathbb{T} \rightarrow \mathbb{T}$ such that

$$
e^{2 \pi i s(\widetilde{f}(x)+C)}=g(x) / g(T x) .
$$

It follows that

$$
\left|\int_{\mathbb{T}} e^{2 \pi i s \widetilde{f}^{\left(q_{n}\right)}(x)} d x\right|=\left|\int_{\mathbb{T}} g(x) / g\left(T^{q_{n}} x\right) d x\right| \rightarrow 1,
$$

contrary to Proposition 3.

Recall now that if $h: \mathbb{T} \rightarrow \mathbb{R}$ is an absolutely continuous function with zero mean, then $h^{\left(q_{n}\right)} \rightarrow 0$ uniformly (see for instance [8] (p. 189)). Consequently, since

$$
\int_{\mathbb{T}} e^{2 \pi i s \widetilde{f}^{\left(q_{n}\right)}(x)} d x-\int_{\mathbb{T}} e^{2 \pi i s(\widetilde{f}+h)^{\left(q_{n}\right)}(x)} d x \rightarrow 0
$$

for all real $s$, and $\left(\widetilde{f}^{\left(q_{n}\right)}\right)_{*} \lambda_{\mathbb{T}} \rightarrow \varrho$ implies $\left((\tilde{f}+h)^{\left(q_{n}\right)}\right)_{*} \lambda_{\mathbb{T}} \rightarrow \varrho$, we obtain the following.

THEOREM 6. If $h: \mathbb{T} \rightarrow \mathbb{R}$ is an absolutely continuous function with zero mean then

- the cylinder flow $T_{\widetilde{f}+h}$ is ergodic,

- if $C>0$ satisfies $\tilde{f}+h+C>0$ then the special flow $T^{\tilde{f}+h+C}$ is weakly mixing and is disjoint from all mixing and all Gaussian flows.

ExAmple 1. Let $f(x)=\log \{x\}$. Then

$$
\begin{aligned}
\widehat{f}[n] & =\int_{0}^{1} e^{2 \pi i n x} \log x d x=\frac{1}{n} \int_{0}^{n} e^{2 \pi i t} \log \frac{t}{n} d t \\
& =\frac{1}{n} \int_{0}^{n} e^{2 \pi i t} \log t d t=O(1 / n)+\frac{1}{n} \int_{1}^{n} e^{2 \pi i t} \log t d t \\
& =O(1 / n)+\frac{1}{n}\left(\left[\frac{1}{2 \pi i} e^{2 \pi i t} \log t\right]_{1}^{n}-\frac{1}{2 \pi i} \int_{1}^{n} e^{2 \pi i t} \frac{1}{t} d t\right) \\
& =O(1 / n)+i O(\log n / n) .
\end{aligned}
$$

Consequently, $f$ satisfies conditions (1)-(4). 
4.1. Spectral disjointness. In this section we deal with the case where $f(x)=-\log \{x\}-1$. We prove that the special flow $T^{\widetilde{f}+C}$ is then also spectrally disjoint from all mixing flows, whenever $\alpha$ satisfies the following condition:

$$
\liminf _{n}\left(\log q_{n}\right) q_{n}\left\|q_{n} \alpha\right\|<\infty .
$$

By passing to a subsequence of $\left(q_{n}\right)$ if necessary we can assume that all $q_{n}$ are even denominators (the reasoning in the case where $q_{n}$ are odd denominators is similar), that

$$
\left(\widetilde{f}^{\left(q_{n}\right)}\right)_{*} \lambda_{\mathbb{T}} \rightarrow \varrho
$$

in $\mathcal{P}(\mathbb{R})$ and that there exists a bounded sequence $\left(\theta_{n}\right)$ of positive numbers such that

$$
\alpha=\frac{p_{n}}{q_{n}}+\frac{\theta_{n}}{\left(\log q_{n}\right) q_{n}^{2}} .
$$

Proposition 7. There exist positive constants $\bar{c}, c$ such that

$$
e^{-s \bar{c}} \leq \varrho(\{t \in \mathbb{R}:|t| \geq s\}) \leq e^{-s c}
$$

for all positive s large enough.

For the proof we will need some lemmas. However let us first record an immediate consequence of Proposition 7.

COROLlary 8. The Fourier transform of the measure @ has an analytic extension to the complex strip $\{z \in \mathbb{C}:|\operatorname{Im} z|<c\}$.

Put $q:=q_{n}$ and $\theta:=\theta_{n}$. Consider the function $f_{q}: \mathbb{T} \rightarrow \mathbb{R}$ given by

$$
f_{q}(x)=-\sum_{j=0}^{q-1} \log \{x+j / q\}-q .
$$

Define

$$
A_{q}=\bigcup_{j=0}^{q-1}[j / q+2\|q \alpha\|,(j+1) / q-2\|q \alpha\|] .
$$

Then $\left|A_{q}\right|=1-4 q\|q \alpha\|$.

Lemma 9. There exists a positive constant $c_{1}$ such that

$$
\left|f^{(q)}(x)-f_{q}(x)\right| \leq c_{1} \quad \text { for all } x \in A_{q} \text { and all } q .
$$

Proof. Let $x \in A_{q}$. Then $x=\left(j_{0}+t\right) / q$, where $0 \leq j_{0}<q$ and $2 q\|q \alpha\| \leq$ $t \leq 1-2 q\|q \alpha\|$. Since in each interval $[j / q,(j+1) / q]$ there exists a unique point of the form $x+k_{j} \alpha, 0 \leq k_{j}<q$, we have 


$$
\begin{aligned}
\left|f^{(q)}(x)-f_{q}(x)\right| & =\sum_{j=0}^{q-1}\left(\log \frac{j+t+k_{j}\|q \alpha\|}{q}-\log \frac{j+t}{q}\right) \\
& \leq \sum_{j=0}^{q-1} \log \left(1+\frac{q\|q \alpha\|}{j+t}\right) \leq q\|q \alpha\| \sum_{j=0}^{q-1} \frac{1}{j+t} \\
& \leq q\|q \alpha\|\left(\frac{2}{t}+\log q\right) \leq 1+\theta,
\end{aligned}
$$

which proves the lemma.

Let $\delta:[0,1) \rightarrow \mathbb{R}$ be defined by

$$
\delta(x)= \begin{cases}x & \text { for } 0 \leq x<1 / 2 \\ 1-x & \text { for } 1 / 2 \leq x<1\end{cases}
$$

LEMMA 10. There exists a positive constant $c_{2}$ such that

$$
-c_{2} \leq-\log \delta(\{q x\})-c_{2} \leq \widetilde{f}_{q}(x) \leq-\log \delta(\{q x\})+c_{2}
$$

for every $x \in \mathbb{T}$ and all $q$.

Proof. First recall that

$$
-\sum_{k=0}^{n} \log (x+k) \leq-\log n !-x \log n+\log \Gamma(x)
$$

for all $x>0$ and $n \in \mathbb{N}$ (see the proof of Theorem 8.19 in [20]). Moreover, by the Stirling formula, there exists $c^{\prime}>0$ such that

$$
-c^{\prime} \leq \log n^{n}+\frac{1}{2} \log 2 \pi n-\log n !-n \leq c^{\prime}
$$

for every natural $n$. Put

$$
\gamma:=\max _{1 / 2 \leq x \leq 3 / 2} \log \Gamma(x) .
$$

Since the functions $\widetilde{f}_{q}$ and $\log \delta(\{q \cdot\})$ are invariant under rotation by $1 / q$ and are even, we only need to show (9) for $0 \leq x \leq 1 /(2 q)$. Now applying (10) and (11) we see that if $0 \leq x \leq 1 /(2 q)$, then

$$
\begin{aligned}
\widetilde{f}_{q}(x)= & -\sum_{j=0}^{q-1} \log (x+j / q)-\sum_{j=1}^{q} \log (-x+j / q)-2 q \\
\leq & -\log x-2 q+2 \log q^{q}+\log 2 \\
& -\sum_{j=1}^{q} \log (q x+j)-\sum_{j=1}^{q} \log (-q x+j)
\end{aligned}
$$




$$
\begin{aligned}
\leq & -\log x-2 q+2 \log q^{q}+\log 2-2 \log (q-1) ! \\
& -(q x+1) \log (q-1)-(-q x+1) \log (q-1) \\
& +\log \Gamma(q x+1)+\log \Gamma(-q x+1) \\
\leq & -\log x-2 q+2 \log q^{q}-2 \log q !+3 \log 2+2 \gamma \\
\leq & -\log x-\log 2 \pi q+2 c^{\prime}+3 \log 2+2 \gamma \\
\leq & -\log q x+2 c^{\prime}+3 \log 2+2 \gamma .
\end{aligned}
$$

Moreover, using (11) we obtain

$$
\begin{aligned}
\widetilde{f}_{q}(x) & =-\sum_{j=0}^{q-1} \log (x+j / q)-\sum_{j=1}^{q} \log (-x+j / q)-2 q \\
& \geq-\log x-2 q-\sum_{j=1}^{q-1} \log \left((j / q)^{2}-x^{2}\right) \geq-\log x-2 q-\sum_{j=1}^{q-1} \log (j / q)^{2} \\
& =-\log x-2 q+2 \log q^{q}-2 \log q ! \geq-\log x-\log 2 \pi q-2 c^{\prime} \\
& \geq-\log q x-\log 2 \pi-2 c^{\prime},
\end{aligned}
$$

which completes the proof.

Proof of Proposition \%. Take $\delta>0$ so that $\varrho\left(\left\{-2 c_{1}-c_{2}-\delta\right\}\right)=0$. By Lemmas 9 and 10,

$$
\begin{aligned}
\varrho\left(\left\{t \in \mathbb{R}: t \geq-2 c_{1}-c_{2}-\delta\right\}\right) & =\lim _{n \rightarrow \infty} \lambda_{\mathbb{T}}\left(\left\{x \in \mathbb{T}: \widetilde{f}^{\left(q_{n}\right)}(x) \geq-2 c_{1}-c_{2}-\delta\right\}\right) \\
& \geq \liminf _{n} \lambda_{\mathbb{T}}\left(\left\{x \in A_{q_{n}}: \widetilde{f}_{q_{n}}(x) \geq-c_{2}\right\}\right) \\
& \geq \lim _{n \rightarrow \infty} \lambda_{\mathbb{T}}\left(A_{q_{n}}\right)=1 .
\end{aligned}
$$

Let $s$ be a real number which is not an atom of $\varrho$ and such that $s>2 c_{1}+$ $c_{2}+\log 2$. Suppose that

$$
x \in \bigcup_{j=0}^{q_{n}-1}\left[\frac{j+e^{-s+2 c_{1}+c_{2}}}{q_{n}}, \frac{j+1-e^{-s+2 c_{1}+c_{2}}}{q_{n}}\right] .
$$

If $2 q_{n}\left\|q_{n} \alpha\right\| \leq e^{-s+2 c_{1}+c_{2}}$, then $x \in A_{q_{n}}$ and

$$
\widetilde{f}^{\left(q_{n}\right)}(x) \leq \widetilde{f}_{q_{n}}(x)+2 c_{1} \leq-\log \delta(\{q x\})+2 c_{1}+c_{2} \leq s,
$$

by Lemmas 9 and 10. Consequently,

$$
\begin{aligned}
\varrho(\{t \in \mathbb{R}: t \leq s\}) & =\lim _{n \rightarrow \infty} \lambda_{\mathbb{T}}\left(\left\{x \in \mathbb{T}: \widetilde{f}^{\left(q_{n}\right)}(x) \leq s\right\}\right) \\
& \geq \lim _{n \rightarrow \infty} \lambda_{\mathbb{T}}\left(\bigcup_{j=0}^{q_{n}-1}\left[\frac{j+e^{-s+2 c_{1}+c_{2}}}{q_{n}}, \frac{j+1-e^{-s+2 c_{1}+c_{2}}}{q_{n}}\right]\right) \\
& =1-2 e^{-s+2 c_{1}+c_{2}} .
\end{aligned}
$$


It follows that there exists $c>0$ such that

$$
\varrho(\{t \in \mathbb{R}: t \geq s\}) \leq 2 e^{-s+2 c_{1}+c_{2}} \leq e^{-c s}
$$

for all $s$ large enough.

Now let $s$ be a real number which is not an atom of $\varrho$ and such that $s>-2 c_{1}-c_{2}+\log 2$. Suppose that $2 q_{n}\left\|q_{n} \alpha\right\| \leq e^{-s-2 c_{1}-c_{2}}$ and

$$
x \in B_{q_{n}}=A_{q_{n}} \cap \bigcup_{j=0}^{q_{n}-1}\left[\frac{j}{q_{n}}, \frac{j+e^{-s-2 c_{1}-c_{2}}}{q_{n}}\right] \cup\left[\frac{j+1-e^{-s+2 c_{1}+c_{2}}}{q_{n}}, \frac{j+1}{q_{n}}\right] .
$$

Then

$$
\widetilde{f}^{\left(q_{n}\right)}(x) \geq \widetilde{f}_{q_{n}}(x)-2 c_{1} \geq-\log \delta(\{q x\})-2 c_{1}-c_{2} \geq s,
$$

by Lemmas 9 and 10 and $\lambda_{\mathbb{T}}\left(B_{q_{n}}\right)=2\left(e^{-s-2 c_{1}-c_{2}}-2 q_{n}\left\|q_{n} \alpha\right\|\right)$. Consequently,

$$
\begin{aligned}
\varrho(\{t \in \mathbb{R}: t \geq s\}) & =\lim _{n \rightarrow \infty} \lambda_{\mathbb{T}}\left(\left\{x \in \mathbb{T}: \widetilde{f}^{\left(q_{n}\right)}(x) \geq s\right\}\right) \\
& \geq \lim _{n \rightarrow \infty} \lambda_{\mathbb{T}}\left(B_{q_{n}}\right)=2 e^{-s-2 c_{1}-c_{2}} .
\end{aligned}
$$

It follows that there exists $\bar{c}>0$ such that

$$
\varrho(\{t \in \mathbb{R}: t \geq s\}) \geq 2 e^{-s-2 c_{1}-c_{2}} \geq e^{-\bar{c} s}
$$

for all $s$ large enough.

Proposition 11. Let $F: \mathbb{T} \rightarrow \mathbb{R}$ be a strictly positive $L^{2}$-function and $\left\{q_{n}\right\}$ be a rigidity time for $T$. If the sequence $\left\{\left\|F^{\left(q_{n}\right)}-q_{n} \int F(x) d x\right\|_{L^{2}}\right\}$ is bounded and a limit distribution @ of the sequence $\left\{\left(F^{\left(q_{n}\right)}-q_{n} \int F(x) d x\right)_{*} \lambda_{\mathbb{T}}\right\}$ has exponential decay of its tails then the special flow $T^{F}$ is spectrally disjoint from all mixing flows.

Proof. The proof is based on the main result in [6], from which it follows that

$$
\left(T^{F}\right)_{C q_{n}} \rightarrow \int_{\mathbb{R}}\left(T^{F}\right)_{-t} d \varrho(t),
$$

where $C=\int F(x) d x$. Now suppose that $g$ is an element of $L_{0}^{2}(X, \mathcal{B}, \mu)$ whose spectral measure $\tau_{g}$ is a Rajchman measure (i.e. the Fourier transform of $\tau_{g}$ vanishes at $\infty$ ). We then have

$$
0=\lim _{n \rightarrow \infty}\left\langle g \circ\left(T^{F}\right)_{C q_{n}}, g\right\rangle=\int_{\mathbb{R}}\left\langle g \circ\left(T^{F}\right)_{-s}, g\right\rangle d \varrho(s) .
$$

Therefore,

$$
\int_{\mathbb{R}} \widehat{\varrho}(u) d \tau_{g}(u)=\int_{\mathbb{R}}\left(\int_{\mathbb{R}} e^{-i s u} d \tau_{g}(u)\right) d \varrho(s)=0 .
$$

By taking any $h$ in the cyclic space of $g$ and repeating the above reasoning we deduce that $\int_{\mathbb{R}} \widehat{\varrho}(u) d \nu=0$ for every finite measure $\nu$ absolutely continuous with respect to $\tau_{g}$. Therefore, $\widehat{\varrho}(\cdot)=0 \tau_{g}$-a.e. On the other hand, by 
Corollary 5 , $\varrho$ has at most a countable number of zeros. Since the measure $\tau_{g}$ is continuous we deduce that $\tau_{g}$ must be the zero measure, so $g$ is constantly equal to zero and the proof is complete.

Let us recall now that if $h: \mathbb{T} \rightarrow \mathbb{R}$ is a function of bounded variation with zero mean, then $\left\|h^{\left(q_{n}\right)}\right\|_{\infty} \leq \operatorname{Var} h$ (see for instance [8, p. 73]). Suppose that $\varrho_{1}$ is a limit distribution of the sequence $\left\{\left((\widetilde{f}+h)^{\left(q_{n}\right)}\right)_{*} \lambda_{\mathbb{T}}\right\}$. By Proposition 7 , the measure $\varrho_{1}$ is not a Dirac measure and its tails decay exponentially. Now an application of Corollary 5.3 in [6] and of Proposition 11 gives the following:

THEOREM 12. If $h: \mathbb{T} \rightarrow \mathbb{R}$ is a function of bounded variation with zero mean and $C>0$ satisfies $\tilde{f}+h+C>0$ then the special flow $T^{\tilde{f}+h+C}$ is disjoint from all Gaussian flows and is spectrally disjoint from all mixing flows.

Finally, an application of Proposition 2 and Theorems 6 and 12 proves Theorem 1.

\section{References}

[1] J. Aaronson, M. Lemańczyk, C. Mauduit and H. Nakada, Koksma's inequality and group extensions of Kronecker transformations, in: Algorithms, Fractals and Dynamics, Y. Takahashi (ed.), Plenum Press, 1995, 27-50.

[2] D. V. Anosov and A. B. Katok, New examples in smooth ergodic theory. Ergodic diffeomorphisms, Trans. Moscow Math. Soc. 23 (1970), 1-35.

[3] S. H. Aranson, Trajectories on nonorientable two-dimensional manifolds, Mat. Sb. 80 (122) (1969), 297-313.

[4] A. A. Blokhin, Smooth ergodic flows on surfaces, Trans. Moscow Math. Soc. 27 (1972), 117-134.

[5] I. P. Cornfeld, S. V. Fomin and Ya. G. Sinaŭ, Ergodic Theory, Springer, New York, 1982.

[6] K. Frączek and M. Lemańczyk, A class of special flows over irrational rotations which is disjoint from mixing flows, Ergodic Theory Dynam. Systems, to appear; available via http://www.mat.uni.torun.pl/preprints/.

[7] H. Furstenberg, Disjointness in ergodic theory, minimal sets and diophantine approximation, Math. Systems Theory 1 (1967), 1-49.

[8] M. Herman, Sur la conjugaison différentiable des difféomorphismes du cercle à des rotations, Publ. Mat. IHES 49 (1979), 5-234.

[9] K. M. Khanin and Ya. G. Sină, Mixing of some classes of special flows over a circle rotation, Funct. Anal. Appl. 26 (1992), 155-169.

[10] A. B. Katok, Constructions in Ergodic Theory, unpublished manuscript.

[11] Y. Khintchin, Continued Fractions, Chicago Univ. Press, 1960.

[12] A. V. Kochergin, On the absence of mixing in special flows over the rotation of a circle and in flows on a two-dimensional torus, Dokl. Akad. Nauk SSSR 205 (1972), 515-518 (in Russian).

[13] —, Time change for flows and mixing, Izv. Akad. Nauk SSSR 37 (1973), 1275-1298 (in Russian). 
[14] A. V. Kochergin, On the mixing of special flows over interval exchange maps and in smooth flows on surfaces, Mat. Sb. 96 (1975), 471-502 (in Russian).

[15] —, Nondegenerate saddles, and the absence of mixing, Math. Notes 19 (1976), 277286.

[16] L. Kuipers and H. Niederreiter, Uniform Distribution of Sequences, Wiley, 1974.

[17] M. Lemańczyk, Sur l'absence de mélange pour des flots spéciaux au-dessus d'une rotation irrationnelle, Colloq. Math. 84/85 (2000), 29-41.

[18] M. Lemańczyk, F. Parreau and D. Volný, Ergodic properties of real cocycles and pseudo-homogeneous Banach spaces, Trans. Amer. Math. Soc. 348 (1996), 49194938.

[19] N. G. Markley, The Poincaré-Bendixson theorem for the Klein bottle, ibid. 135 (1969), 159-165.

[20] W. Rudin, Principles of Mathematical Analysis, McGraw-Hill, 1976.

[21] K. Schmidt, Cocycles of Ergodic Transformation Groups, Lect. Notes in Math. 1, Mac Millan Co. of India, 1977.

Faculty of Mathematics and Computer Science

Nicolaus Copernicus University

Chopina 12/18

87-100 Toruń, Poland

E-mail: fraczek@mat.uni.torun.pl

mlem@mat.uni.torun.pl

Received 9 May 2003;

in revised form 12 January 2004 